\title{
Placebo-Induced Changes in Spinal Cord Pain Processing
}

\author{
Dagfinn Matre, ${ }^{1,2,3}$ Kenneth L. Casey, ${ }^{2,3}$ and Stein Knardahl ${ }^{1,4}$ \\ ${ }^{1}$ Department of Physiology, National Institute of Occupational Health, N-0033 Oslo, Norway, ${ }^{2}$ Neurology Research Laboratory, Veterans Affairs Medical \\ Center, and ${ }^{3}$ Department of Neurology, University of Michigan, Ann Arbor, Michigan 48105, and ${ }^{4}$ Department of Psychology, University of Oslo, N-0317 \\ Oslo, Norway
}

\begin{abstract}
Pain is an essential sensory modality, signaling injury or threat of injury. Pain perception depends on both biological and psychological factors. However, it is not known whether psychological factors modify spinal mechanisms or if its effect is limited to cortical processing. Here, we use a placebo analgesic model to show that psychological factors affect human spinal nociceptive processes. Mechanical hyperalgesia (hypersensitivity) after an injury is attributable to sensitized sensory neurons in the spinal cord. After a $5 \mathrm{~min}, 46^{\circ} \mathrm{C} \mathrm{heating}$ of the skin, subjects developed areas of mechanical hyperalgesia. This area was smaller in a placebo condition compared with a baseline condition. This result suggests that placebo analgesia affects the spinal cord as well as supra-spinal pain mechanisms in humans and provides strong supporting evidence that placebo analgesia is not simply altered reporting behavior. Central sensitization is thought to mediate the exaggerated pain after innocuous sensory stimulation in several clinical pain conditions that follow trauma and nervoussystem injury. These new data indicate that expectation about pain and analgesia is an important component of the cognitive control of central sensitization.
\end{abstract}

Key words: descending; spinal cord; pain; sensitization; nociception; inhibition

\section{Introduction}

The present study sought to determine whether psychological factors like placebo suggestions can affect spinal nociceptive mechanisms. Pain is an essential sensory modality, signaling injury or threat of injury. Pain perception depends on both biological and psychological factors. Central nervous mechanisms in the spinal cord and in the brain amplify or inhibit nociceptive signals. Psychological factors like attention, appraisal of threat, expectations, attitudes, and pain beliefs modify pain perception. Placebo analgesia is one example of psychological factors that reduce pain and is associated with decreased neural activity in pain-sensitive brain regions (Petrovic et al., 2002; Wager et al., 2004). Increased activity during anticipation of pain in the midbrain region of the periacquaductal gray area, which contains systems that inhibit pain, indicates that expectations alter inhibitory systems (Wager et al., 2004).

The present study tested the hypothesis that psychological processes involved in placebo analgesia modify spinal mechanisms of central sensitization. A human model sensitizing spinal cord dorsal horn neurons by heating the skin was tested (LaMotte et al., 1991; Cervero et al., 1993). This produces an area of increased sensitivity, surrounding the injured area, that is hypersensitive to punctuate stimuli (termed hyperalgesia) and to stroking touch (termed allodynia) (LaMotte et al., 1991; Cervero et al., 1993; Warncke et al., 1997).

Received 0ct. 4, 2005; revised Nov. 22, 2005; accepted Nov. 23, 2005

This work was supported by grants from the Norwegian Research Council (D.M.) and the United States Department of Veteran's Affairs (K.L.C.). We are grateful to all of the volunteer participants.

Correspondence should be addressed to Dr. Dagfinn Matre, National Institute of Occupational Health, P.0. Box 8149 Departement, N-0033 0slo, Norway. E-mail: Dagfinn@stami.no.

DOI:10.1523/JNEUROSCI.4218-05.2006

Copyright $\odot 2006$ Society for Neuroscience $\quad$ 0270-6474/06/260559-05\$15.00/0
Previous studies in the rat have shown that blocking descending nerve traffic from the brain stem to the spinal cord may enhance sensitization in response to intense electrical stimulation of an afferent nerve (Gjerstad et al., 2001). Hence, it seems that descending traffic may modify nociceptive mechanisms in the spinal cord. We tested whether a placebo condition reduces the hyperalgesic skin area relative to the hyperalgesic area that develops under a baseline condition with identical temperature. This should be supportive evidence for a placebo-induced inhibition of spinal pain pathways.

\section{Materials and Methods}

Subjects. Sixty-four healthy volunteers, who responded to ads in a major newspaper and to flyers posted at universities and colleges in Oslo, Norway, were included in the study. All subjects reported good health. They were instructed not to drink alcohol the last $48 \mathrm{~h}$ before the experiment and not to drink coffee the day of the experiment. None of the subjects took any prescription drugs, and they were instructed to discontinue use of any over-the-counter analgesics at least $12 \mathrm{~h}$ before each experimental day.

Twenty-nine subjects successfully completed the entire study. Ten subjects were dismissed after session 1 because they did not develop mechanical hyperalgesia after a $5 \mathrm{~min}, 46^{\circ} \mathrm{C}$ heating (see below, Induction and quantification of hyperalgesia). Four subjects were dismissed after session 1 because of too large areas on the volar forearm that were insensitive to warmth (tested by touching different spots on the forearm for $3 \mathrm{~s}$ with the thermode heated to $40^{\circ} \mathrm{C}$ ) (Green and Cruz, 1998). Twenty-one subjects were dismissed after session 2 because of large dayto-day variability $(>25 \%$ increase or decrease in pinprick hyperalgesic area). After the exclusions, 19 subjects remained in the placebo group (age range, 20-44 years; nine females) and 10 subjects remained in the control group (age range, $20-45$ years; three females). Female subjects were studied on days 12-23 after their last menstruation (Riley et al., 1999). The experimental protocol was conducted in accordance with the 
Helsinki declaration and was approved by the Regional Ethical Committee in Oslo.

Induction and quantification of hyperalgesia. Hyperalgesia was induced by heating the skin of the medial volar forearm to $46^{\circ} \mathrm{C}$ for $5 \mathrm{~min}$. Heat was delivered by a $25 \times 50 \mathrm{~mm}$ Peltier thermode (Thermotest; Somedic AB, Hörby, Sweden) strapped to the forearm. Similar methods have been used in several reports to study the mechanisms of primary and secondary hyperalgesia (LaMotte et al., 1991; Cervero et al., 1993; Warncke et al., 1997; Dirks et al., 2003).

During heat stimulation, the subject rated the intensity continuously on a $100 \mathrm{~mm}$ electronic visual analog scale (VAS) labeled with 0 ("no sensation"), 40 ("pain threshold"), and 100 ("worst pain imaginable"). The area of brush allodynia was determined by gently stroking the skin with a cotton tip along eight linear radials around the stimulation site, starting well outside the presumed hyperalgesic area (Warncke et al., 2000). The heated area was not tested. The strokes were delivered every $3-4 \mathrm{~s}$ at $5 \mathrm{~mm}$ intervals. The subject was looking in another direction during testing. The subject was instructed to alert the experimenter when two subsequent stimulations were clearly more intense than the previous one. The skin was marked, and the process was repeated from another direction. After testing eight directions, the marks were transferred to plastic foil and joined to form an area. To determine the size of the area, the marked area was cut out of the plastic foil and weighed on a highsensitive scale (PB303DR; Mettler, Toledo, Greifensee, Switzerland) in which $10 \mathrm{mg}=0.73 \mathrm{~cm}^{2}$. The area of punctuate hyperalgesia was quantified in the same way using a Von Frey filament $\left(84.4 \mathrm{~g} / \mathrm{mm}^{2}\right.$ pressure).

A stimulus-response curve to three Von Frey hairs of three different intensities $\left(5,50\right.$, and $\left.100 \mathrm{~g} / \mathrm{mm}^{2}\right)$ was then obtained by pressing each hair four times at each of four small areas $\left(\sim 1 \mathrm{~cm}^{2}, 1.5 \mathrm{~cm}\right.$ outside the edge of the primary hyperalgesic area) in a pseudo-random order (Magerl et al., 1998). The subject indicated the perceived intensity by verbally expressing a number on the VAS, and the average rating for each Von Frey intensity was calculated as the mean of the four ratings. Finally, the mechanical pinprick pain threshold was tested with an electronic Von Frey meter with a $1 \mathrm{~mm}^{2}$ probe (Somedic AB, Hörby, Sweden). The probe was pressed perpendicular to the skin at each of the four $1 \mathrm{~cm}^{2}$ areas until the subject indicated that the pressure was painful. The pain threshold was calculated as the mean of the four digitized readings from the meter.

Blood pressure, measured as mean arterial pressure (MAP), and heart rate (HR) were monitored continuously by the Penãz method with a cuff placed on the left third finger (Finapres; Ohmeda, Englewood, CO). MAP and HR signals were sampled $(200 \mathrm{~Hz})$ and analyzed off-line. Oneminute average values of MAP and HR were calculated right before heating and during the last minute of heating.

Instructions to subjects. The advertisement to which placebo subjects responded said that the aim of the study was to test the analgesic effectiveness of a magnet against heat pain. This information was repeated in the laboratory before the first trial. Subjects were shown an example of the magnet to be used $(20 \times 14 \times 1.5 \mathrm{~cm}$; Matrixmagnetics, McKinney, TX). The company had, after our instructions, made an ordinary magnet and a sham magnet that looked identical. The sham magnet was used in all of the experiments. Placebo subjects were told that four baseline trials would be performed on the first 2 days and that the magnet would be tested during two trials on session 3. During session 3, the information about the analgesic magnet was repeated to reinforce the subject's expectations of heat analgesia. The advertisement to which control group subjects responded said that the aim of the study was to investigate hypersensitivity of the skin after a heat stimulus. They were also told that four baseline trials would be performed on the first 2 days and two temperatures would be tested during session 3 . They were told that the first trial during session 3 would be made at $43^{\circ} \mathrm{C}$ and that the second trial would be made at the baseline temperature of $46^{\circ} \mathrm{C}$. They were not told anything about magnets, but to make the physical conditions identical in the two groups, they were told that a metal plate (the sham magnet) with a built-in thermometer would be strapped alongside the thermode during session 3 to measure heat dissipation to the surroundings.

Study design. The study included three experimental sessions, each lasting $\sim 1 \mathrm{~h}$ and taking place $4-7 \mathrm{~d}$ apart (Fig. 1). During each session,

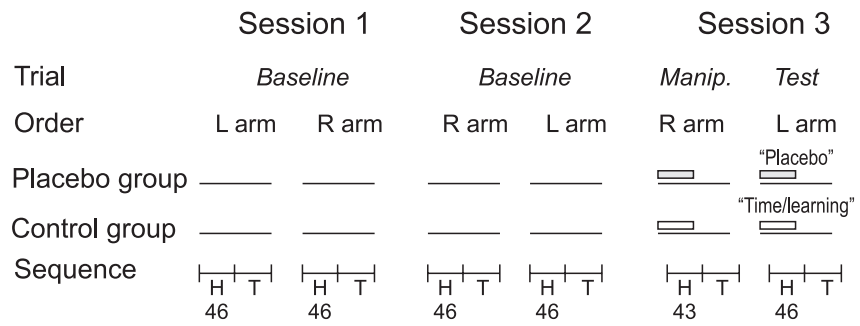

$\varpi / \sqsubset$ Sham magnet attached during heating. Introduced as "magnetic analgesia" in placebo group and "measurement of heat dissipation" in control group

$\mathrm{H}$ - heat stimulation at 43 or $46^{\circ} \mathrm{C}$

$\mathrm{T}$ - sensory testing

Order - opposite in half of subjects Session 1 and 2, randomized Session 3

Figure 1. The experiment was performed over three sessions (separated by $4-7 \mathrm{~d}$ ) and six trials. In each trial, hyperalgesia was induced by applying a 5 min contact heat stimulus $(H)$ to the skin of the volar forearm, immediately followed by sensory testing $(T)$. The first four trials (sessions 1 and 2) were identical baselines, and heat was delivered at $46^{\circ} \mathrm{C}$. During session 3 , the sham magnet was strapped alongside the thermode during heating. Placebo subjects were told it was a pain-relieving magnet (shaded rectangles). Control subjects were told it was measuring heat dissipation to the surroundings (open rectangles). In the manipulation (Manip.) trial, the temperature was lowered to $43^{\circ} \mathrm{C}$. The placebo subjects were not told that the intensity was reduced. Hence, during the subsequent test trial, placebo subjects expected magnetic pain relief. Because the heat was again $46^{\circ} \mathrm{C}$ (as during baseline), this became the placebo condition. Contrary to the placebo subjects, control subjects were informed about the reduced intensity during manipulation. Hence, control subjects did not expect pain relief during the test trial, which became a control condition for time and learning. L, Left; $R$, right.

hyperalgesia was induced and quantified on both volar forearms as described above (i.e., six trials). During session 3, the sham magnet was strapped alongside the thermode during heating (without interfering with the contact between the thermode and the skin). During the first trial on session 3 ("manipulation" trial), the temperature was reduced to $43^{\circ} \mathrm{C}$. Placebo subjects were not informed that the temperature was reduced (Montgomery and Kirsch, 1997; Price et al., 1999). The aim with this conditioning procedure was to increase the expectancy of analgesia before the next trial, the test trial, in which the temperature again was $46^{\circ} \mathrm{C}$. Control subjects were informed that the temperature was reduced during this trial. Consequently, they did not expect analgesia during the final "test" trial.

The "placebo effect" was assessed by comparing test trial outcomes with "baseline" trial outcomes from the corresponding arm in the placebo group. The effects of time and learning ("time/learning effect") were assessed in the same manner from the control group. The effect of manipulation was also determined. For each outcome measure, the effect size was calculated as the mean for the control group minus the mean for the placebo group divided by the pooled within-group $\mathrm{SD}, d=\left[\bar{x}_{\text {control }}\right.$ $\left.-\bar{x}_{\text {placebo }}\right]$ /pooled SD (Vase et al., 2002). One-half of the subjects started with the left arm, and one-half of the subjects started with the right arm. During session 2, the order was reversed. During session 3, the order was randomized. The ambient temperature was $23-24^{\circ} \mathrm{C}$. Exactly the same information was given to all of the subjects within each group. The investigator was the same person in all sessions. After the final session, the experimenter informed the participants in the placebo group about the true aim of the study.

Statistics. Unless noted otherwise, Friedman nonparametric ANOVA was used to compare the outcome measures with the three experimental sessions as factor. In case of a main effect $(p<0.05)$, the Wilcoxon signed rank test was used for paired comparisons. A repeated-measures ANOVA was used to compare MAP and HR measurements because these measures are considered to be normally distributed.

\section{Results}

Reduced heat-pain intensity during placebo

A significant placebo effect was found on the heat-pain intensity (Fig. 2A, left). The median VAS score was reduced from $46 \mathrm{~mm}$ 

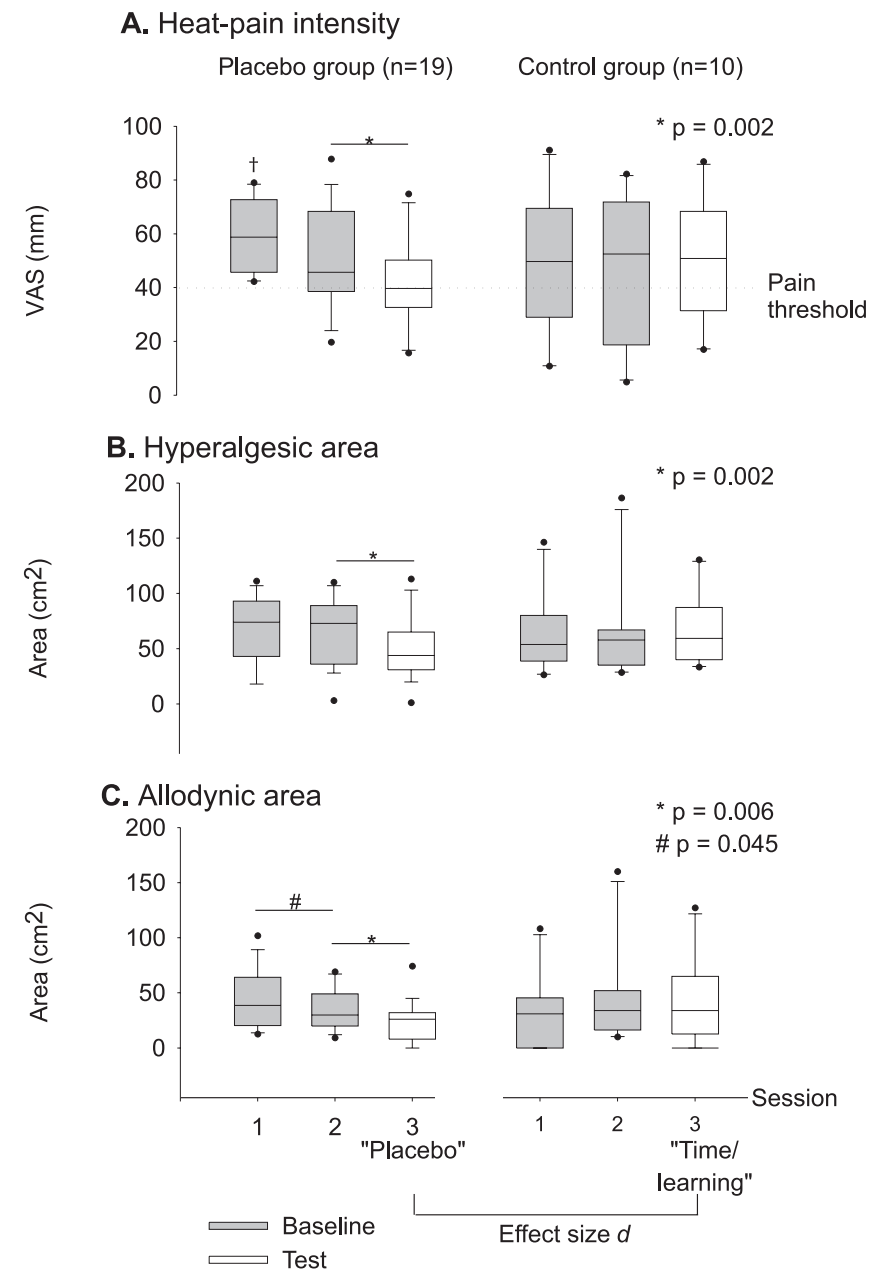

Figure 2. $\quad A$, A significant placebo effect was found on heat-pain intensity ratings (left). No effect was found of time and learning (right). $B, C$, A significant placebo effect was found on hyperalgesic and allodynic areas (left). No effect was found of time and learning (right). Data are displayed as the median, interquartile range (box), 5-95\% confidence intervals (leaf), and outliers (dots). ${ }^{\dagger}$ Session 1 ratings were not included in the statistical comparison because only 9 (of 19) ratings were obtained during this session.

during baseline (session 2) to $40 \mathrm{~mm}$ during the test, with $79 \%$ of the participants showing an effect in this direction $(Z=-3.1$; $p=0.002)$. No time/learning effect was found in the control group (Fig. $2 A$, right); the median VAS score was 56 and $55 \mathrm{~mm}$ during the second baseline and test trials, respectively $\left(\chi^{2}=0.6\right.$; $p=0.74)$. Comparing VAS scores between groups during the test trial gave an effect size $d$ of 0.74 in favor of the placebo group.

\section{Smaller hyperalgesic and allodynic areas during placebo}

A placebo effect was found on the hyperalgesic areas (Fig. $2 B$, left). The median hyperalgesic area was reduced from 73 to 44 $\mathrm{cm}^{2}(Z=-3.1 ; p=0.002)$, with $89 \%$ of the participants showing an effect in this direction. No time/learning effect was found in the control group $\left(\chi^{2}=0.46 ; p=0.79\right)$ (Fig. $2 B$, right). The effect size $d$ for the hyperalgesic areas during the test was 0.53 in favor of the placebo group. A placebo effect was also found on the allodynic areas (Fig. $2 C$, left). The median allodynic area was reduced from 34.5 to $26 \mathrm{~cm}^{2}(Z=-2.75 ; p=0.006)$, with $74 \%$ of the participants showing an effect in this direction. No time/learning effect was found in the control group $\left(\chi^{2}=5.43 ; p=0.066\right)$ (Fig. $2 C$, right). The effect size $d$ for the allodynic areas during the test was 1.02 in favor of the placebo group.

\section{Unchanged tactile sensitivity}

The two additional measures of cutaneous sensitivity obtained from the secondary hyperalgesic area, (1) tactile sensitivity to pinprick stimuli of increasing intensity and (2) pinprick pain thresholds, were not changed between sessions (i.e., unaffected by placebo and time/learning). VAS ratings of pinprick stimuli and pinprick pain thresholds, as well as test statistics, are shown in Table 1.

\section{Unchanged blood pressure and HR}

The MAP and HR (Table 2) did not differ across the three experimental sessions in any of the groups or when comparing before heating to the end of heating (Table 3). It was hypothesized that, on an individual basis, subjects with a large increase in blood pressure from before heating to the end of heating would show a more prominent reduction in heat-pain intensity and consequently a reduction in hyperalgesic areas. Spearman's correlation coefficient was calculated for the relationship between these outcome measures and changes in blood pressure, but none of these correlations were significant (Table 4).

\section{Comparing baseline sessions}

No differences were found between baseline sessions (sessions 1 and 2 ) for the hyperalgesic areas in either group $(p>0.79)$. In the placebo group, the allodynic area was slightly smaller during session 2 compared with session $1(p=0.045)$, whereas for the control group, there was no difference ( $p=0.26$ ) (Fig. $2 C$, left).

\section{Discussion}

Our results show that, during the placebo condition, the development of mechanical secondary hyperalgesia is less pronounced than during baseline. As expected, there was a placebo effect on reported pain during the induction process when the magnet was present. However, there was also a placebo effect on the area of secondary hyperalgesia, suggesting that placebo mechanisms are modulating the induction of hyperalgesia in addition to the perception of heat pain during induction.

In this experiment, the placebo object (the magnet) was present only during the induction of mechanical hyperalgesia. A transfer of the placebo effect from heat-pain intensity to the area of hyperalgesia and allodynia is highly unlikely, because subjects were led to expect only reduced heat intensity. Because the area of hyperalgesia is determined by intensity-dependent spinal segmental mechanisms, our results suggest that the placebo effect was expressed at the spinal cord as well as at supra-spinal levels. To our knowledge, this is the first time a placebo response is associated with spinal pain processing in humans. It should provide a strong argument against allegations that placebo analgesia is nothing but altered reporting behavior (Hrobjartsson and Gotzsche, 2001).

Placebo analgesia is generated by information that modifies expectancies and appraisal of sensations, in addition to factors that influence the reporting of pain (Price et al., 1999). These factors, in addition to conditioning and descending modification of spinal nociceptive pathways, modify pain. In the present study, a combination of suggestion and possibly conditioning were probably important factors in generating an expectation of pain relief. Suggestion of "magnetic heat-pain relief" was given in the advertisement and was repeated by the investigator before and during the procedure. Additionally, we sought to maximize the authority of the suggestions by referring to studies in which magnets had proven effective. The magnet served as a conditioned stimulus being presented with lower heat pain. The procedure of 
surreptitiously reducing the stimulus intensity before testing has been used in several placebo studies (Montgomery and Kirsch, 1997; Price et al., 1999; Wager et al., 2004) and has been shown to be more powerful than verbal expectancy (suggestion) alone (Voudouris et al., 1990).

Although the placebo object was present only during heat, it may be argued that the reduction of hyperalgesic areas during placebo stems from a transfer of the placebo effect from the heating phase to the sensory-testing phase. Such an explanation cannot totally be ruled out, but we find this highly unlikely. First, during the final test trial, the magnet had only been used in one trial at $43^{\circ} \mathrm{C}$ (manipulation) as opposed to four trials at $46^{\circ} \mathrm{C}$. The removal of the magnet before sensory testing should prevent associations of the sham magnet to testing of the area of hyperalgesia. Second, we suggested that the magnet was effective against heat pain, not against mechanical pain. Third, subjects showed no change in response to the Von Frey and pinprick testing in the affected area (Table $1)$.

Although the hyperalgesic areas can be considered objective measures of spinal neuronal sensitization, we measured the subjects' report of these areas. It may be argued that the subjects altered their reporting behavior during the placebo-effect testing (by stopping the mechanical testing later and thereby creating a smaller area) either because they realized that we expected a reduced area by a "social contract" (Wager, 2005) or because of a transfer of the placebo effect on heat pain to the situation of testing the hyperalgesic area. Importantly, the subjects were not able to see their forearm during the testing. Whether the subject is able to recognize the "manipulated area" as being smaller depends on their ability to discriminate between two tactile stimulations (two-point discriminability threshold). At the forearm, this threshold is $\sim 38 \mathrm{~mm}$ (Weinstein, 1968). In the present data, there are eight linear differences for each subject between the placebo and baseline conditions (one difference for each of the test directions). In $99.1 \%$ of the cases, the change (from baseline to placebo) was below the $38 \mathrm{~mm}$ threshold for spatial discrimination. Hence, it would not be possible for the entire group of subjects to have created the smaller hyperalgesic areas during placebo by either conscious or subconscious mechanisms.

The neurophysiological mechanisms mediating the reduced hyperalgesic area during placebo in this study cannot be determined. In animal studies, increased descending traffic inhibits nociceptive mechanisms in the spinal cord (Gjerstad et al., 2001). Our data suggest that these corticospinal and bulbospinal pathways may mediate placebo analgesia in humans, an explanation that would be compatible with previous hypotheses of spinal nociceptive modulation (Melzack and Wall, 1965). This explanation is also consistent with observations from monkey, in which attentional manipulations decrease the activity in some trigeminothalamic dorsal horn neurons (Duncan et al., 1987), indicating that descending pathways are responsible for filtering and adjusting sensory information.

Placebo analgesia has been found to be associated with increased activity in the orbitofrontal cortex, a structure that has
Table 3. Statistical summary comparing HR and MAP with two-way, repeatedmeasures ANOVA (sessions 1-3 as one factor and before-after as one factor)

\begin{tabular}{llllll}
\hline & \multicolumn{2}{l}{ Main-effect session } & & \multicolumn{2}{l}{ Main effect before versus end } \\
& $F$ & $p$ & & $F$ & $p$ \\
\hline HR (bpm) & 1.346 & 0.285 & & 3.937 & 0.079 \\
$\quad$ Control group & 0.11 & 0.898 & & 0.024 & 0.885 \\
$\quad$ Placebo group & & & & \\
MAP (mmHg) & 0.751 & 0.486 & & 0.099 & 0.76 \\
$\quad$ Control group & 1.391 & 0.281 & & 1.391 & 0.281 \\
$\quad$ Placebo group & & & & \\
\hline
\end{tabular}

Table 4. Spearman's correlation coefficients (Rho) testing the relationship between the difference in MAP (after minus before heating) against standardized changes in hyperalgesic area, allodynic area, and heat-pain intensity between sessions 2 and 3

\begin{tabular}{|c|c|c|c|c|c|c|}
\hline & \multicolumn{2}{|c|}{ Hyperalgesic area } & \multicolumn{2}{|c|}{ Allodynic area } & \multicolumn{2}{|c|}{ Heat-pain intensity } \\
\hline & Rho & $p$ & Rho & $p$ & Rho & $p$ \\
\hline \multicolumn{7}{|l|}{ Correlations } \\
\hline Control group & 0.122 & 0.737 & -0.45 & 0.31 & 0.453 & 0.189 \\
\hline Placebo group & -0.361 & 0.141 & -0.105 & 0.689 & 0.307 & 0.215 \\
\hline
\end{tabular}

been related to cognitive pain modulation (Rainville et al., 1999; Petrovic et al., 2002; Kringelbach and Rolls, 2004). Wager et al. (2004) dissociated the period of pain from the expectation of pain and found a correlation between expectation and increased activity in the prefrontal cortex, an area associated with the maintenance of information needed for cognitive control. Furthermore, during placebo, they observed increased activity near the periaqueductal gray. The periaqueductal gray has been identified as a site for control of pain modulation in animals (Basbaum and Fields, 1984) and humans (Tracey et al., 2002) and provides a link to the descending pain-modulatory system. A consequence of inhibition of central sensitization in the spinal medulla during placebo would be reduced activation in brain areas responding to nociception. Hence, based on the present data, it is possible that 
reduced nociceptive afferent signaling contributes to the reduced activation reported in brain regions such as the thalamus, insula, and anterior cingulate cortex during placebo (Wager et al., 2004).

To summarize, the placebo-induced reduction in the area of hyperalgesia and allodynia was not a "demand characteristic" (Wager, 2005) because (1) the placebo object (sham magnet) was absent during sensory testing, (2) subjects received no instructions relating "magnet" effects to the area of hyperalgesia or allodynia, (3) the subjects were not able to see their forearm during testing, and (4) the average placebo effect was below the average spatial resolution of the forearm. Because the area of hyperalgesia is determined by intensity-dependent spinal segmental mechanisms, our results suggest that the placebo effect was expressed at the spinal cord as well as at supra-spinal levels. Central sensitization is considered to be an explanation for the exaggerated pain after innocuous sensory stimulation in several clinical pain conditions that follow trauma or injury to the nervous system (Desmeules et al., 2003; Banic et al., 2004). In conclusion, the present study indicates that the development of central sensitization is partly under cognitive control.

\section{References}

Banic B, Petersen-Felix S, Andersen OK, Radanov BP, Villiger PM, ArendtNielsen L, Curatolo M (2004) Evidence for spinal cord hypersensitivity in chronic pain after whiplash injury and in fibromyalgia. Pain 107:7-15.

Basbaum AI, Fields HL (1984) Endogenous pain control systems: brainstem spinal pathways and endorphin circuitry. Annu Rev Neurosci 7:309-338.

Cervero F, Gilbert R, Hammond RG, Tanner J (1993) Development of secondary hyperalgesia following non-painful thermal stimulation of the skin: a psychophysical study in man. Pain 54:181-189.

Desmeules JA, Cedraschi C, Rapiti E, Baumgartner E, Finckh A, Cohen P, Dayer P, Vischer TL (2003) Neurophysiologic evidence for a central sensitization in patients with fibromyalgia. Arthritis Rheum 48:1420-1429.

Dirks J, Petersen KL, Dahl JB (2003) The heat/capsaicin sensitization model: a methodological study. J Pain 4:122-128.

Duncan GH, Bushnell MC, Bates R, Dubner R (1987) Task-related responses of monkey medullary dorsal horn neurons. J Neurophysiol 57:289-310.

Gjerstad J, Tjolsen A, Hole K (2001) Induction of long-term potentiation of single wide dynamic range neurones in the dorsal horn is inhibited by descending pathways. Pain 91:263-268.

Green BG, Cruz A (1998) "Warmth-insensitive fields": evidence of sparse and irregular innervation of human skin by the warmth sense. Somatosens Mot Res 15:269-275.

Hrobjartsson A, Gotzsche PC (2001) Is the placebo powerless? An analysis of clinical trials comparing placebo with no treatment. N Engl J Med 344:1594-1602.

Kringelbach ML, Rolls ET (2004) The functional neuroanatomy of the human orbitofrontal cortex: evidence from neuroimaging and neuropsychology. Prog Neurobiol 72:341-372.

LaMotte RH, Shain CN, Simone DA, Tsai EF (1991) Neurogenic hyperalgesia: psychophysical studies of underlying mechanisms. J Neurophysiol 66:190-211.

Magerl W, Wilk SH, Treede RD (1998) Secondary hyperalgesia and perceptual wind-up following intradermal injection of capsaicin in humans. Pain 74:257-268.

Melzack R, Wall PD (1965) Pain mechanisms: a new theory. Science 150:971-979.

Montgomery GH, Kirsch I (1997) Classical conditioning and the placebo effect. Pain 72:107-113.

Petrovic P, Kalso E, Petersson KM, Ingvar M (2002) Placebo and opioid analgesia-imaging a shared neuronal network. Science 295:1737-1740.

Price DD, Milling LS, Kirsch I, Duff A, Montgomery GH, Nicholls SS (1999) An analysis of factors that contribute to the magnitude of placebo analgesia in an experimental paradigm. Pain 83:147-156.

Rainville P, Hofbauer RK, Paus T, Duncan GH, Bushnell MC, Price DD (1999) Cerebral mechanisms of hypnotic induction and suggestion. J Cogn Neurosci 11:110-125.

Riley JL, Robinson ME, Wise EA, Price DD (1999) A meta-analytic review of pain perception across the menstrual cycle. Pain 81:225-235.

Tracey I, Ploghaus A, Gati JS, Clare S, Smith S, Menon RS, Matthews PM (2002) Imaging attentional modulation of pain in the periaqueductal gray in humans. J Neurosci 22:2748-2752.

Vase L, Riley III JL, Price DD (2002) A comparison of placebo effects in clinical analgesic trials versus studies of placebo analgesia. Pain 99:443-452.

Voudouris NJ, Peck CL, Coleman G (1990) The role of conditioning and verbal expectancy in the placebo response. Pain 43:121-128.

Wager TD (2005) Expectations and anxiety as mediators of placebo effects in pain. Pain 115:225-226.

Wager TD, Rilling JK, Smith EE, Sokolik A, Casey KL, Davidson RJ, Kosslyn SM, Rose RM, Cohen JD (2004) Placebo-induced changes in FMRI in the anticipation and experience of pain. Science 303:1162-1167.

Warncke T, Stubhaug A, Jorum E (1997) Ketamine, an NMDA receptor antagonist, suppresses spatial and temporal properties of burn-induced secondary hyperalgesia in man: a double-blind, cross-over comparison with morphine and placebo. Pain 72:99-106.

Warncke T, Stubhaug A, Jorum E (2000) Preinjury treatment with morphine or ketamine inhibits the development of experimentally induced secondary hyperalgesia in man. Pain 86:293-303.

Weinstein S (1968) Intensive and extensive aspects of tactile sensitivity as a function of body part, sex, and laterality. In: The skin senses (Kenshalo DR, ed), pp 195-222. Springfield, IL: Thomas. 